\title{
MOLECULAR CYTOGENETICS
}

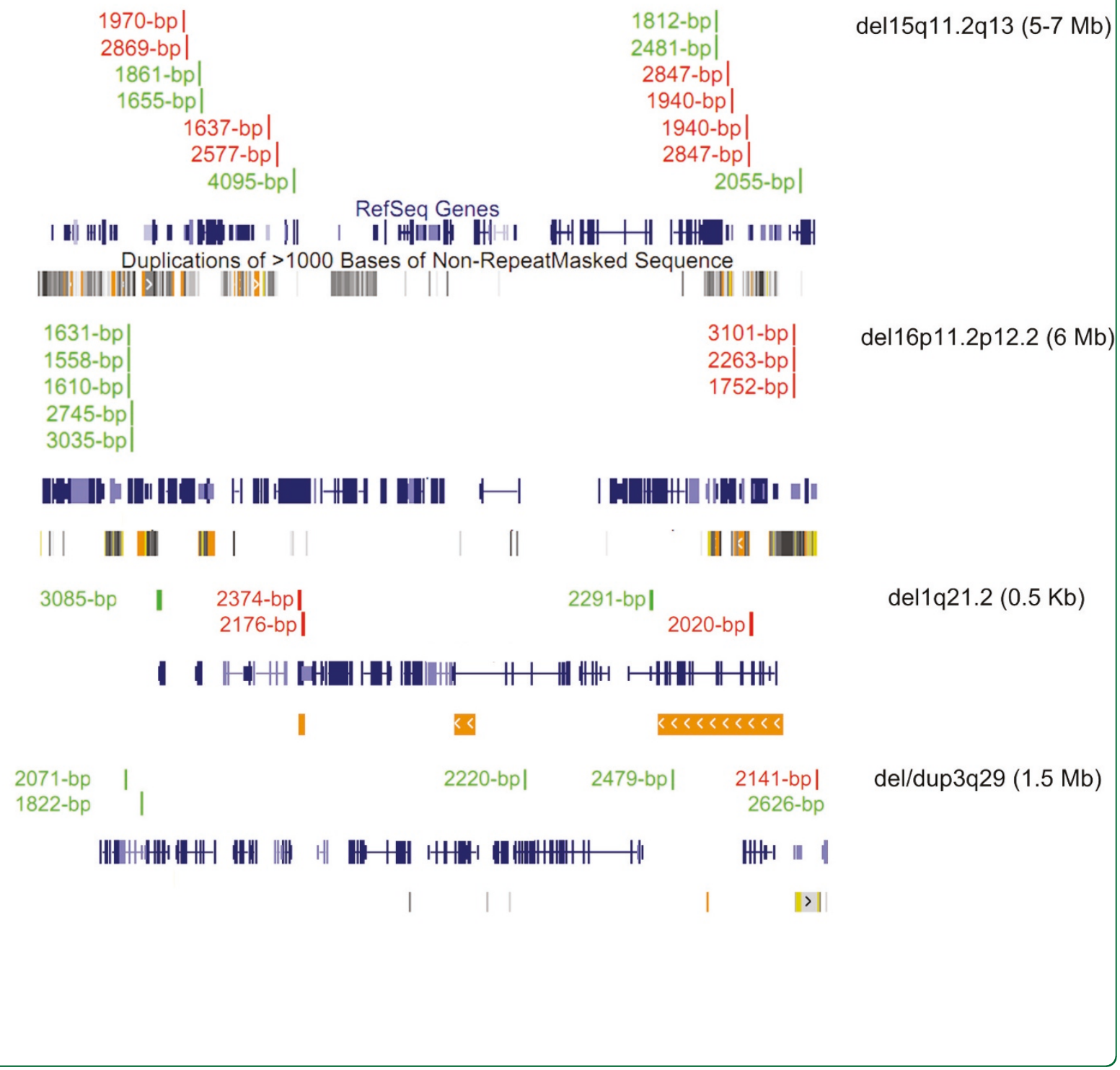

\section{Context-based FISH localization of genomic rearrangements within chromosome 15q11.2q13 duplicons}

Khan et al. 


\title{
Context-based FISH localization of genomic rearrangements within chromosome 15q11.2q13 duplicons
}

Wahab A Khan ${ }^{1,2}$, Joan HM Knoll ${ }^{2}$ and Peter K Rogan ${ }^{1,3^{*}}$

\begin{abstract}
Background: Segmental duplicons (SDs) predispose to an increased frequency of chromosomal rearrangements. These rearrangements can cause a diverse range of phenotypes due to haploinsufficiency, in cis positional effects or gene interruption. Genomic microarray analysis has revealed gene dosage changes adjacent to duplicons, but the high degree of similarity between duplicon sequences has confounded unequivocal assignment of chromosome breakpoints within these intervals. In this study, we localize rearrangements within duplicon-enriched regions of Angelman/Prader-Willi (AS/PWS) syndrome chromosomal deletions with fluorescence in situ hybridization (FISH).

Results: Breakage intervals in AS deletions were localized recursively with short, coordinate-defined, single copy (SC) and low copy (LC) genomic FISH probes. These probes were initially coincident with duplicons and regions of previously reported breakage in AS/PWS. Subsequently, probes developed from adjacent genomic intervals more precisely delineated deletion breakage intervals involving genes, pseudogenes and duplicons in 15q11.2q13. The observed variability in the deletion boundaries within previously described Class I and Class II deletion AS samples is related to the local genomic architecture in this chromosomal region.
\end{abstract}

Conclusions: Chromosome 15 abnormalities associated with SDs were precisely delineated at a resolution equivalent to genomic Southern analysis. This context-dependent approach can define the boundaries of chromosome rearrangements for other genomic disorders associated with SDs.

\section{Introduction}

The human genome contains numerous regions that exhibit rare structural chromosome rearrangements due to segmental duplicons (SDs) that predispose to recurrent genomic disorders [1,2]. SDs are composed of large $(10 \mathrm{~kb}-400 \mathrm{~kb})$ near identical (> 95\%) paralogs of DNA, that are found at physically distinct genomic locations and can include genes and pseudogenes [3]. There are at least 20 distinct genomic sites in the human genome flanked by duplicons implicated in recurrent pathogenic rearrangements [3]. Among these are deletions of chromosome 15q11.2q13 in Angelman (AS [MIM 105830]) and Prader-Willi syndromes (PWS [MIM 176270]). AS

\footnotetext{
* Correspondence: progan@uwo.ca

'Department of Biochemistry, University of Western Ontario, Laboratories of Genome Bioinformatics and Genomic Disorders, 1151 Richmond Street, London, ON, Canada

Full list of author information is available at the end of the article
}

and PWS share two common size classes of de novo deletions that differ in proximal extent of the deletion [4]. Class I ( $\sim 7 \mathrm{Mb})$ and Class II $(\sim 5 \mathrm{Mb})$ deletions have variable genomic lengths and span from proximal to distal breakpoints 1 (BP1) to 3 (BP3) and breakpoints 2 (BP2) to 3 (BP3), respectively [5,6]. Large SDs containing sequences in the HERC2 gene family (Hect Domain and RLD2 [MIM 605837]), arising by transposition to the proximal and distal ends of chromosome $15 \mathrm{q} 11.2 \mathrm{q} 13$, have been localized to the BP1, BP2 and BP3 hotspots $[7,8]$. Additional genomic architectures containing GOLGA8E-associated SDs (golgin subfamily a, $8 \mathrm{E}$ ) can catalyze rearrangements between $15 \mathrm{q} 11$ and 15q24q26 [9].

Deletions in chromosome 15q11.2q13 have been characterized with custom-designed DNA microarrays and confirmed by fluorescence in situ hybridization (FISH) using BAC clones [10-13]. Breakage activity within

\section{Biomed Central}


highly homologous duplicons, however, is challenging to ascertain with techniques such as array CGH alone, because interpretation of context-independent hybridization data is confounded by the presence of multiple closely related, non-contiguous SDs [14].

The aim of this study was to delineate SDs at the boundaries of 15q11.2q13 deletions by FISH using individual sequence-defined, short-target single copy (SC) and low copy (LC) DNA probes $[15,16]$. LC probes occur in 2 to 10 copies in the haploid genome. Genomic coordinate-defined SC-FISH has been used for diagnosis of congenital and acquired disorders [15,16], including, for example, definition of an atypical microdeletion in Smith-Magenis Syndrome (SMS [MIM 182290]) [16]. Similarly designed SC probes, composed of tiled sets of oligonucleotides, spanning larger targets have also been used to detect chromosomal abnormalities [17]. In the present study, SC and LC probes are embedded within and adjacent to SD sequences. A set of 15q11.2q13 LC probes and adjacent SC probes were developed to interrogate Class I and Class II AS deletions on metaphase chromosomes. Using sequential hybridizations of LC and SC FISH probes, it is possible to determine which duplicon intervals have been retained or have been disrupted in AS deletions.

\section{Methods \\ Categorization of breakage of documented AS/PWS deletions}

Deletion boundaries in AS/PWS, determined previously by BAC [10-12] and oligonucleotide arrays [13], were reviewed and annotated using the UCSC genome browser [http://genome.ucsc.edu/, hg18 or NCBI 36 assembly]. These boundaries were approximated from both recombinant BAC sequences [18] and oligonucleotide probe genome coordinates [14] that showed reduced copy number. These data were used to locate the BP1, BP2 and BP3 breakage regions in Class I and Class II deletions $[5,7,8]$. Five breakage sub-intervals denoted in Figure 1 (Regions A through E) are centromeric to BP1 (CEN-BP1), within BP1, BP2, and BP3, respectively. Their corresponding genome coordinates are chr15:18,683,000-18,980,000 for region A (CEN-BP1); $18,980,000-20,385,000$ for region B (BP1); 20,385,000$20,700,000$ for region $C$ (BP1-BP2); 20,700,000$21,356,000$ for region D (BP2); and 25,941,000$27,286,000$ for region E (BP3). Breakage intervals have also been annotated for 15q13.2q13.3 deletions within BP4 and BP5 (19) [not shown in Figure 1].

\section{Defining genomic SC and LC intervals}

Genomic SC and LC sequences, ranging from 1500 to $5000 \mathrm{bp}$ in length (per chromosome target), were batchprocessed using the Galaxy metaserver http://main.g2. bx.psu.edu/[20]. The coordinates of these sequences in custom browser tracks derived from 15q11.2q13 were intersected with the approximate locations of documented breakpoints inferred from BAC and oligonucleotide array CGH [10-13]. SC and LC intervals overlapping and adjacent to these breakage intervals were prioritized for FISH probe design. These tracks were also used to determine the locations of SC and LC intervals relative to known SDs in 15q11.2q13 (Regions A-E). LC intervals lacking repetitive sequences (red) within blocks of SDs were identified and sorted from SC intervals (green) that mapped adjacent to duplicon structures (Figure 1C). The presence of these SC and LC intervals was confirmed in the Reference and alternate (HuRef, Celera) genome assemblies. BLAST analysis showed 100\% sequence identity of SC intervals and 90-99\% identity of paralogous LC intervals among the different assemblies.

As copy number variants (CNVs) bracketed by duplicon structures can confound interpretation of FISH data, we selected only those probes with the highest sequence identity for their genomic locus and observed in the expected copy number (Figure 2A). The majority of $\mathrm{CNVs}$ in this region (typically $>1 \mathrm{Mb}$ in $\mathrm{BP} 1, \mathrm{BP} 2$ and BP3) completely overlapped both SC and LC probe sequences. The presence of a polymorphic duplication separated by large genomic distances (> 5-6 Mb), coincident with SC or LC probes, in an AS deletion is expected to result in a non-contiguous hybridization pattern. There was no evidence of this pattern in our results, which is not surprising in light of the low frequency of these CNVs in the population [21]. Nevertheless, the presence of CNVs in the $15 \mathrm{q} 11.2 \mathrm{q} 13$ region associated with SDs should be considered in genomic coordinate-defined SC and LC probe designs.

\section{Probe development and fluorescence in situ hybridization (FISH)}

Primer3 [http://frodo.wi.mit.edu/primer3/[22] was used to design oligonucleotide primer pairs for $6 \mathrm{SC}$ and 4 LC intervals of which one SC probe was from the common deletion region and served as a positive control (Additional File 1, Table S1). SC and LC genomic intervals were amplified using long PCR [23] with the Platinum ${ }^{\circledR} P f x$ DNA polymerase kit (Invitrogen ${ }^{\mathrm{TM}} \mathrm{CA}$, USA). PCR conditions were optimized by gradient thermal cycling. Amplicons were purified using the QIAquick gel extraction kit (Qiagen ON, Canada), labeled by nick translation with biotin-dUTP or digoxigenin-dUTP (Roche Diagnostics, ON, Canada) and detected with avidin-FITC or Cy3-conjugated digoxin antibody [24]. Probes were validated on metaphase chromosomes from at least 2 normal lymphocyte cytogenetic preparations (including one male) following approval by the Office of Research Ethics at the University of Western Ontario. 
Probes were analysed for chromosome location and hybridization pattern as deduced from the Human Genome Reference sequences. At least 20 metaphase cells were scored for each probe and a hybridization efficiency of $\geq 80 \%$ was required to qualify a probe.

\section{AS cell lines}

Six AS lymphoblastoid cell lines were characterized in this study. The cell lines were previously determined to have either Class I (WJK36, WJK67, WJK70) or Class II deletions (WJK18, WJK24, WJK35) by DNA analysis [5]. The cell lines were blind coded until FISH analysis was complete. FISH with SC and LC probes were performed with either one probe/one color or two probe/two color detection to delimit the boundaries of the rearrangement within or adjacent to15q11.2q13 duplicons. Twenty to 50 metaphase cells were examined per probe for each AS cell line. Cells were imaged using an automated epifluorescence microscopy system (Metasystems Inc, MA). 


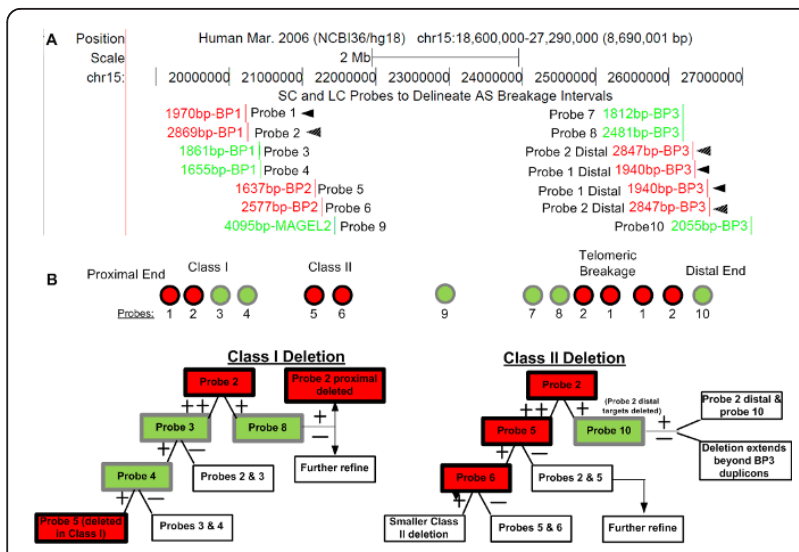

Figure 2 SC and LC Probes Developed for FISH. A) High resolution map of LC (red) and SC (green) probes. Proximal targets of probes 1 and 2 (arrowheads) in BP1 share 99\% sequence identity to their distal BP3 targets (arrowheads). Probes 3, 4, 7, 8 and 10 are adjacent to duplicon regions. LC probes 5 and 6 mimic SC probes as each have one paralog target in BP2 and their other paralogs are divergent and distal of 15q11.2q13 or interchromosomal (see text).

Probe 9 serves as a deletion control that targets a sequence $\sim 20 \mathrm{~Kb}$ centromeric of MAGEL2. Probe lengths are reported in base pairs (bp). B) Metaphase FISH algorithm for delineating Class I and Class II deletions in 15q11.2q13. Probes 1 through 10 are color-coded. A schema of hybridization experiments is shown for Class I and II deletions. Different outcomes indicated by the presence $(+)$ or absence (-) of an SC (green) or LC probe (red) prescribes the procedure for delineating boundaries of a breakage interval (unfilled box). If two paralogous LC targets are retained; this is indicated by a '++' symbol. Further refinement of a deletion interval requires either sequential application of additional SC probes or dual-color/dualprobe hybridization.

\section{Results}

\section{Selected SC and LC probes and their relationship to genomic architecture}

Our strategy selected LC FISH probes within duplicons to interrogate rearrangements at both ends of chromosome $15 \mathrm{q} 11.2 \mathrm{q} 13$. SC probes, adjacent to either the proximal or distal duplicons targeted by an LC probe, were then hybridized to establish whether the proximal or distal LC target was deleted. LC intervals selected for probe development were based on: 1) their location in or adjacent to a region of frequent documented breakage; 2) up to 3 chromosome 15q11.2q13 targets detected by the LC probe; and 3) genomic separation of $\geq 5 \mathrm{Mb}$ for at least 2 of $3 \mathrm{LC}$ probe targets with chromosome $15 q 11.2 q 13$. LC probe targets separated by less than 5 $\mathrm{Mb}$ could not be unequivocally discriminated by FISH as distinct loci on metaphase chromosomes. Hybridization patterns of LC probes with one target within $15 q 11.2 q 13$ and diverse sequence targets elsewhere in the genome were scored in a similar manner to an SC probe.

After bioinformatic analysis, 40 SC and LC intervals were marked for potential development of FISH probe reagents (Figure $1 \mathrm{C}$ ). Nine of these intervals were selected based on the algorithm described below. They comprised $4 \mathrm{LC}$ and $5 \mathrm{SC}$ regions (see Figure 2A for probe map and designation). Probe details (centromere to telomere) are described below. LC probes 1 and 2 are embedded within duplicons common to BP1 and BP3. Both LC probe intervals in BP1 (probe 1, $1970 \mathrm{bp}$; and probe 2, $2869 \mathrm{bp)}$ are coincident with breakage sites inferred from oligonucleotide arrays [13] and proximal to breakage sites inferred from BAC microarrays [10-12]. The LC probe intervals in BP3 (probe 1, 1940 $\mathrm{bp}$; and probe $2,2837 \mathrm{bp}$ ) are centrally located in the distal region of highest documented breakage activity (Figure 1B, C). SC probes were developed from within BP1 (probe 3, $1861 \mathrm{bp}$; probe 4, $1655 \mathrm{bp}$ ) and within BP3 (probe 7, $1812 \mathrm{bp}$; probe 8, $2481 \mathrm{bp)}$ ). Probes 3 and 4 are adjacent to the duplicons in BP1 that are detected by LC probes 1 and 2 . SC probes 7 and 8 are adjacent to duplicons in BP3 that contain probe 1 and 2 paralogs. These SC probes respectively mark the breakage boundaries within BP1 and BP3. The LC intervals in BP2 (probe 5, $1637 \mathrm{bp}$; and probe 6, $2577 \mathrm{bp}$ ) have homology to duplicons in $15 \mathrm{q} 14$ and $13 \mathrm{q} 31.3$, with approximately $90 \%$ sequence identity over intervals less than $1500 \mathrm{bp}$ in length. The degree of sequence divergence and sizes of these paralogous targets limit the detection of hybridization of probes 5 and 6 to the $15 \mathrm{q} 14$ and 13q31.3 loci. Therefore, LC probes 5 and 6 mimic the hybridization patterns of SC probes in the BP2 region. $\mathrm{SC}$ probe 10 (2055 bp) hybridizes to a target from within APBA2 [MIM 602712]) which is distal of BP3, and detects larger deletions [19]. SC probe 9 (4095 bp) is a positive control for AS/PWS deletions and maps $\sim 20 \mathrm{~Kb}$ centromeric of MAGEL2 [MIM 605283]).

\section{FISH algorithm}

FISH studies with SC and LC probes validated the bioinformatic analysis. These probes can delineate boundaries of a chromosomal rearrangement in a single hybridization for certain breakpoints, however improved chromosome resolution was achieved through recursive hybridization using a series of probes. LC probes that detect fewer duplicon targets than anticipated from the genomic architecture; represent a deletion of one or more paralogs. Subsequent FISH analysis with adjacent $\mathrm{SC}$ probes from $\mathrm{BP} 1$ or $\mathrm{BP} 3$ can then determine which of the LC probe targets has been deleted. The process can be expedited by co-hybridizing the initial LC probe with a differentially-labeled SC probe adjacent to one of the duplicons. Figure 2B illustrates the strategy in which individual SC and LC probes are selected for FISHbased assays. The initial probe selected for hybridization varies depending upon whether the deletion has been previously classified. The order of probes used for 
subsequent hybridizations depends on the results of the previous FISH experiment. Both Class I and II deletions were analyzed with LC probe 2 , since it targets a larger genomic interval than LC probe 1 , and provides information about sequences found at both ends of the $15 q 11.2 q 13$ deletion. Figure 3A shows that probe 2 hybridizes to separate duplicons in BP1 (chr15:20,241, 611-20,244,479) and BP3 (chr15:26,321,705-26,324,542 and 26,550,044-26,552,881) in normal chromosomes. In AS, duplicon targets within BP1 or BP3 may be deleted. Class I breakage intervals are localized with probes 3 (proximal), 7, 8 and 10 (distal; Figure $3 \mathrm{E}$ and $3 \mathrm{~F}$ ). In Class II deletions, probes 5 and 10 are used for the analysis. If necessary, the Class I breakpoint can be further refined with probes 3 and 4 , and Class II cases with probes 5 (Figure 3B) and 6.

\section{Definition of breakage intervals in AS cell lines}

Prior to undertaking this study, breakage intervals at the ends of the deletions in these AS cell lines were not precisely known, and the rearranged genes and SD features coincident with deletion boundaries had not been characterized.

\section{Class I deletion characterization}

Variability in both the proximal and distal breakage intervals were observed in the three Class I AS cell lines (Figure 4A) such that each deletion differed in size. Deletion of BP3 targets in WJK36 and WJK70 resulted in single locus hybridization to the BP1 target (Figure $3 C$ ), whereas WJK67 was not deleted for either of these targets. The proximal breakage interval was localized within a $161 \mathrm{~Kb}$ interval in WJK67 and WJK70 (Figure $3 \mathrm{D}$; between probes 2 and 3; chr15:20,244,48020,405,122). The distal breakpoint in BP3 of WJK67 was delimited by a $136 \mathrm{~Kb}$ region bounded by probe 7 and one of the probe 2 duplicon targets (chr15:26,184,974$26,321,705$, Figure 4A). In WJK36, the proximal breakage interval in BP1 was defined within a $57.6 \mathrm{~Kb}$ interval (spanning chr15:20,419,289-20,476,942), based on results showing probes 3 and 4 to be intact (Figure 4A) and a previously demonstrated deletion of D15S18 [5]. The lengths of the Class I deletions were approximately 5.78 $\mathrm{Mb}$ (WJK67), 6.07 Mb (WJK36), and 6.14 Mb (WJK70).

\section{Class II deletion characterization}

The deletion breakage interval in BP3 was found to vary among different AS cell lines, with an overall distribution similar to those observed for Class I deletions (Figure 4A). At the centromeric end, chromosomal breaks in Class II AS deletions coincide with a cluster of duplicons in BP2. Based on hybridization results using probes 4 and 5, all cell lines (WJK18, WJK24, and WJK35) exhibited a common proximal deletion breakage interval (Figure 4A). This is consistent with previous microarray analyses indicating that the probe 5 sequence maps to a highly active region of rearrangement (Figure 1B, C). The proximal breakage interval is localized to a $\sim 697.3$ $\mathrm{Kb}$ region within $\mathrm{BP} 2$, since D15S18 is intact [5] in these cell lines (chr15:20,477,088-21,174,481). The distal BP3 breakage interval in WJK35 (Class II) is the same as the one defined in WJK67 (Class I) localized by a deletion of SC probe 7 (Figure 3E). WJK18 and WJK24 shared the same breakage interval in BP3 that was present in WJK70 and WJK36. Probe 10 was intact in cell lines WJK18, WJK24, WJK70, and WJK36 (Figure 3F), thereby refining the breakage interval to a $569 \mathrm{~Kb}$ region (chr15:26,552,881-27,122,231;Figure 4A). The Class II deletions ranged in size from 5.01(WJK35) to $5.38 \mathrm{Mb}$ (WJK18 and WJK24).

\section{Discussion}

We demonstrate that chromosome 15q11.2q13 deletions can be delineated using combinations of single and lowcopy, sequence-defined FISH probes. The LC probes detect SDs, which are prone to rearrangement. Our analysis of these rearrangements distinguishes genes and pseudogenes at the boundaries of deletions that are either deleted or disrupted in AS.

\section{Duplicon architecture of Class I and II AS deletions}

In this study, Class I and Class II breakage intervals coincide with HERC2-containing SDs [7,11,25]. The proximal (103.6 Kb; coordinates: 20.2-20.3 Mb, BP1) and distal SDs (106 Kb; coordinates: 26.5-26.72 Mb, BP3) coincident with breakage intervals in WJK70 (Class I) are distinct and inversely oriented to the paralogous SDs that define breakage intervals in Class I WJK67 (Figure 4B-I, 4B-II). By contrast, in WJK36 (Class I), a more complex pattern was found. The proximal breakage interval was adjacent to the BP1 duplicons, rather than within them (Figure 4B-I). Atypical breakage intervals that fall outside of the duplicon blocks have been reported both in SMS and 16p11.2p12.2 deletions [26]. The breakage intervals in BP3 for WJK36 and WJK70 overlap the same duplicon (Figure 4B-II). Distinct blocks of SDs in BP2 mediate Class II deletion rearrangements (Figure 4B-III), which are respectively paralogous to different sets of duplicon blocks within BP3 (Figure 4BIV). In each cell line, the proximal and distal duplicons at each end of the deletion shared 98\% - 99\% sequence identity.

We and others have found that 15q11.2q13 duplicons and breakage intervals in AS are in some instances coincident [12]. However, this was not the case for WJK67, WJK36 and WJK35, where breakage intervals (Figure 4A) were distinct from previously reported SDs [12]. The proximal duplicons that are rearranged in these individuals are comprised of pericentromeric HERC2 pseudogene sequences and the distal copy contains both 

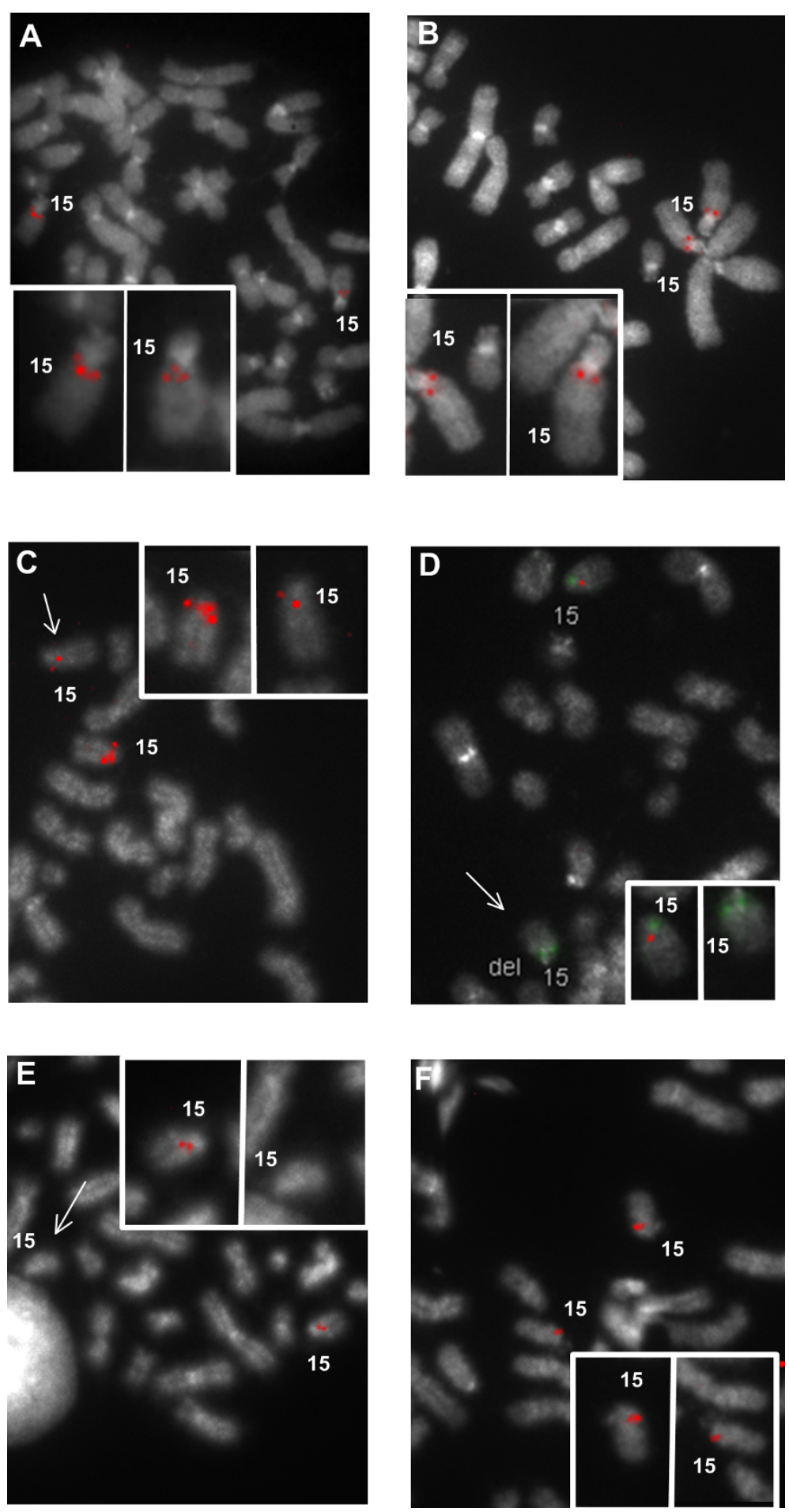

Figure 3 Representative metaphase cell FISH images of SC and LC probes. A) Hybridization pattern in a normal cell of LC probe 2 with targets in BP1 (2869 bp) and BP3 (2837 bp) to both chromosome 15s. B) Hybridization of LC probe 5 (1637 bp) on normal chromosomes. The pattern is similar to that of an SC probe, as the paralogous target of probe 5 exhibits high sequence divergence and is not detected. The next 4 panels show chromosome hybridizations on cells of Class I (C, D) and Class II (E, F) AS deletions. C) LC probe 2 hybridization pattern in WJK36 cell. Only the BP3 target is deleted in the abnormal chromosome (arrow), as determined by subsequent hybridization with SC probe 4 (not shown). The normal chromosome shows hybridization to all paralogous targets. Loss of the BP3 target sequence with this probe was also evident in WJK 70 (Class I), WJK18 and WJK24 (Class II). D) Dual-color hybridization with LC Probe 2 (green) and SC probe 3 (1861 bp, red) in WJK67 (Class I). Probe 2 is intact on both chromosomes and was confirmed by sequential hybridization. Probe 3 is deleted on the abnormal homolog (arrow), and a similar outcome for this probe was noted for WJK70. E) Deletion of SC probe 7 (1812 bp) (arrow) in WJK35. Deletions of probe 7 were also seen in WJK67, WJK70 and WJK36. F) SC probe 10 (2055 bp) is intact in WJK24. Similar hybridization patterns were seen in WJK70, WJK36 and WJK18. All probes were labeled with digoxigenin or biotin- dUTP and detected with Cy-3 digoxin antibody or FITC-avidin, respectively. Cells are counterstained with DAPI. 


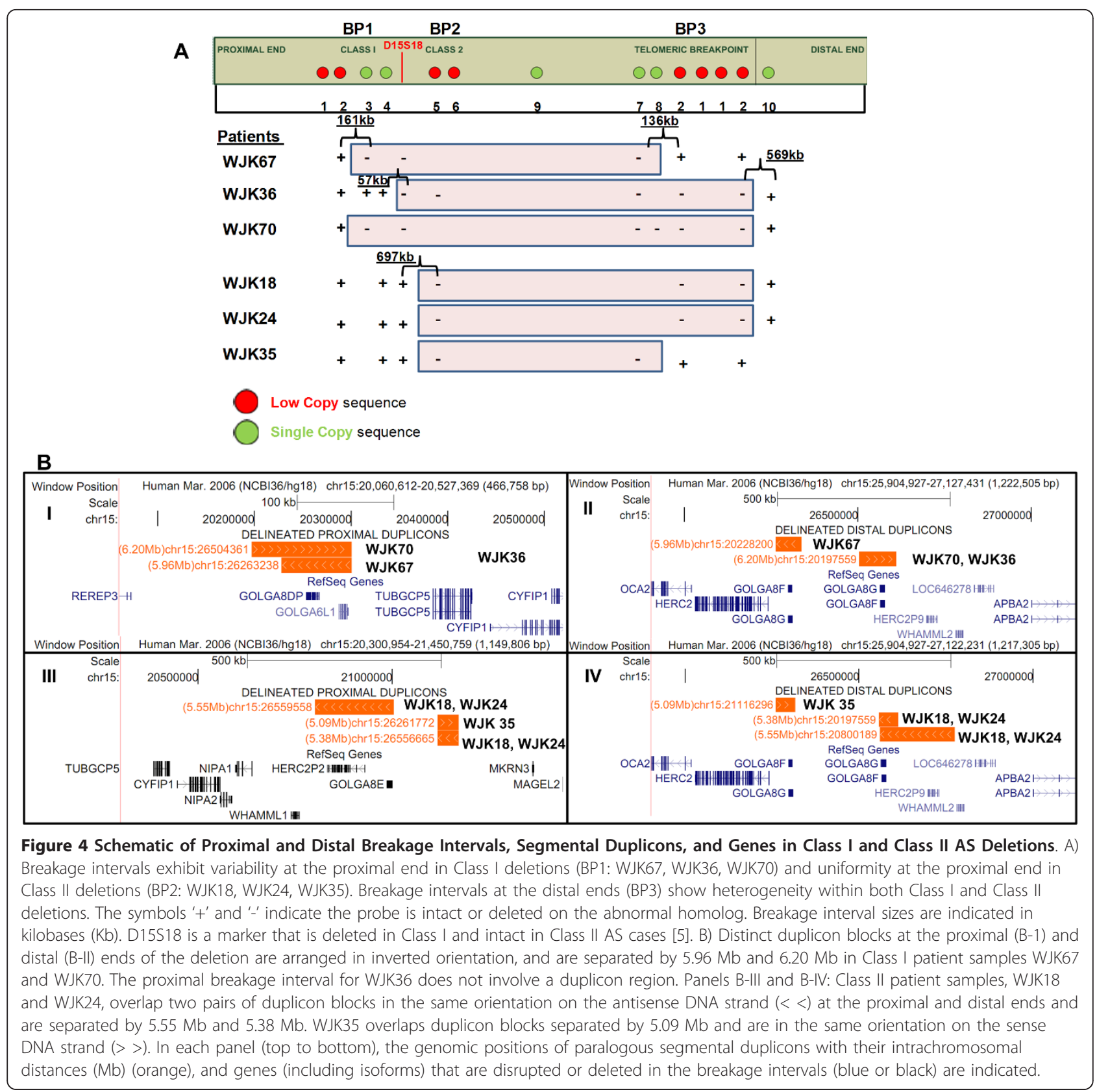

the HERC2 gene and additional HERC2 pseudogenes $[8,27]$. Apparent non-allelic homologous recombination between these HERC2-related duplicons results in diverse breakage intervals with variable length deletions.

Based on the results of this study, the present FISH strategy can be further streamlined. The proximal breakage interval within Class I deletion samples can be delineated by co-hybridization with LC probe 2 and SC probe 3. Deletion of the BP3 duplicon interval can be simultaneously detected with probe 2 . To expedite refinement of Class II deletion breaks, probe 5 in the BP2 duplicon cluster is co-hybridized with probe 8 in
BP3. The combination of probes 4 (BP1) and 5 (BP2) can delineate the deletion class when it is unknown. A Class I deletion is indicated if both of these probes are hemizygous. A Class II deletion is diagnosed if probe 4 is retained and probe 5 is deleted. The additional SC intervals $(n=40$; Figure $1 C)$ we have designed can be used to refine the breakage sites within BP1 and BP2.

\section{Relating breakage intervals to genes}

Contextual mapping of 15q11.2q13 genomic rearrangements bracketed by SDs can distinguish genes that are disrupted from those that are have been demonstrated 
to be deleted using methods that quantify copy number, i.e. array CGH, qPCR, or MLPA. CYFIP1 [MIM 606322] was deleted (Figure 4B-I) and a partial deletion of TUBGCP5 was likely (tubulin, gamma complex associated protein 5 [MIM 608147]) in WJK67 and WJK70. In contrast, the $57 \mathrm{~Kb}$ breakage interval of WJK36 overlapped the 3' boundary of the TUBGCP5 and the 5' region of CYFIP1, effectively disrupting both genes (Figure 4B-I). TUBGCP5 maps between BP1 and BP2 and encodes a protein that is required for microtubule nucleation at the centrosome $[28,29]$. CYFIP1 is associated with FMRP [30], which is implicated in neurite extension, guidance and branching [31]. NIPA2 [MIM 608146]) and NIPA1 [MIM 608145]), which do not overlap any duplicons, were deleted in all Class I cell lines. Dominant mutations in NIPA1 cause hereditary spastic paraplegia [32].

The golgin subfamily genes, GOLGA8DP (golgi autoantigen, golgin subfamily a, 8D) and GOLGA6L1 (golgi autoantigen, golgin subfamily a, 6-like 1), are embedded within SDs. Both of these genes are likely to be disrupted or deleted in WJK67 and WJK70, but are intact in WJK36 (Figure 4B-1). GOLGA6L1 is expressed and predicted to encode a functional protein, whereas $G O L$ GA8DP is currently designated as a pseudogene [33] and has paralogy to duplicons at 15q11.2q13, 15q24 and $15 q 26[9,25]$. In the Class II deletions - WJK18, WJK24 and WJK35, the breakage interval in BP2 coincides with HERC2P (Hect domain and RLD2 pseudogene 2) and the GOLGA8E gene. Both GOLGA8E and HERC2P in BP2 are likely to be disrupted (Figure 4B-III). GOLGA8E is expressed and encodes cDNAs with an open reading frame [9].

Breakage intervals delineated in WJK67 and WJK35 overlap HERC2, whereas these intervals are telomeric in the other AS cell lines. This results in the deletion of $H E R C 2$, as well as potential deletion or disruption of GOLGA8G, GOLGA8F and WHAMML2 (Figure 4B-II, 4D-IV). GOLGA8G and GOLGA8F are expressed pseudogenes [33]. Disruption of functional HERC2 in BP3 can juxtapose it with distal HERC2-related sequences, producing novel fusion transcripts [27]. As expected, OCA2 ([MIM 611409]) was deleted in all cell lines $[5,34,35]$ and $A P B A 2$, which is distal to the telomeric HERC2 cluster in BP3, was intact. Large AS deletions that include $A P B A 2$ in $15 q 13.1$ have been reported [12] and a small duplication of 15q13.1 has been described in a family with a history of autism [36].

\section{Sequence-defined FISH in other duplicon-rich genomic domains}

Sequence-defined SC and LC FISH probes will be generally useful to delineate genomic disorders bracketed by SDs that juxtapose during chromosome rearrangement.
In AS/PWS, SMS, and DiGeorge syndromes, the distribution of SDs enables LC probes to be designed that simultaneously interrogate sequence copy number at both ends of the chromosomal deletion. However, as seen in $15 \mathrm{q} 11.2 \mathrm{q} 13$, some SDs may contain remote paralogous targets that do not participate in the rearrangement. These intervals can still be useful as LC probes, with the remote paralogs serving as hybridization controls in metaphase FISH.

Recently, recurrent microdeletions have been described in chromosomes 16p11.2p12.2 (chr16: 21.3$29.5 \mathrm{Mb}$ ) [26,37], 1q21.1 (chr1: 144.10-144.60 Mb), and a reciprocal deletion/duplication in 3q29 [Ref. [3] for review]. At the centromeric and telomeric breakpoints within $16 \mathrm{p} 11.2$ and 16p12.2 respectively, we identified 3 LC and 5 SC intervals, suitable as FISH probes, co-localizing with breakage activity in this region [26,37]. By combining these SC probes with different sets of centromeric LC probes, the boundaries of $16 \mathrm{p} 11.2 \mathrm{p} 12.2$ rearrangements can be defined.

At the chromosome 1q21.1 and 3q29 loci [3], our analysis showed that duplicons flanking the common deletion interval are too closely spaced to resolve as separate hybridization signals on metaphase chromosomes. Nevertheless, these duplicon blocks can still be assayed by metaphase FISH with LC probes detecting a second interchromosomal or remote intrachromosomal locus. Generally, SC and LC FISH probes can be employed either individually or as an ensemble to analyze both large $(>5 \mathrm{Mb})$ and small $(0.5 \mathrm{~Kb})$ recurrent genomic rearrangements flanked by SDs. The increased resolution of SC and LC FISH in complex duplicon genomic regions may prove useful in distinguishing rearrangements that appear to be similar in length based on array CGH analysis, which actually span overlapping sequences that vary in length.

Interphase SC and LC FISH analysis may be feasible, if multiple, closely-spaced duplicon-related signals from an LC probe originate from the same homolog. We have not yet tested this possibility, given the highly decondensed state of interphase chromatin and the limited knowledge about spatial organization of homologous SDs during interphase.

\section{Comparison with array CGH in duplicon-rich genomic intervals}

Breakage intervals of chromosomal rearrangements associated with SDs have been refined with several array CGH platforms [38-41]. BAC arrays identify the initial aberration and are refined by 2 or more customized oligonucleotide arrays. Breakpoint junctions are then delineated by long PCR or real time PCR with multiple primer pairs staggered along a duplicon block with at least one member of each pair lying in a repeat-masked 
sequence. Most customized oligonucleotide arrays require at least five adjacent oligonucleotide probes to be deleted or duplicated for reliable genotyping [42]. In regions containing a high degree of homology between duplicons, this strategy can result in false-positive signals from probes identical to those within the true microdeletion or microduplication [39].

Therefore, microarray designs generally avoid or provide low probe coverage in intervals that have genomic architectures such as SDs that confound the interpretation of copy number differences $[43,44]$. In relating the breakage intervals to the duplicon architecture and genes, we noted probes in the Agilent SurePrint 244K microarray [13] (Figure 1B) that were present in duplicons with paralogous sequences on other chromosomes and within segmentally duplicated sequences in the BP1, BP2, and BP3 regions (Table 1). On chromosome 15, the cross-hybridizing oligonucleotide probes are on average $\sim 20 \mathrm{~kb}$ apart and are organized in clusters which coincide with SDs in BP1, BP2 and BP3. Since these probe sequences had been expected to be unique, their hybridization to interchromosomal and remote intrachromosomal duplicons would be expected to distort the interpretation of chromosomal deletion boundaries.

The AS breakage intervals were also compared to the corresponding probe coverage by the Affymetrix SNP 6.0 microarray. Generally, there is a paucity of SNP probes within SDs. The breakage intervals delineated by SC and LC probes in the present study are not covered by any probes on the SNP array. However, several divergent SDs within BP1, BP2, and BP3 contained 3-10 probes targeting SNPs within duplicons. These probes also overlapped interchromosomal duplicons (Table 2). We further examined the subset of these SNP probes ( $\mathrm{n}$ = 14) representing the highest degree of cross-hybridization to $15 \mathrm{q} 11.2 \mathrm{q} 13$ breakpoint hotspots and found that 6 contained the same polymorphic variant targeting intra or interchromosmal SDs with $96-100 \%$ similarity to one another.

Table 1 Number of Agilent $244 \mathrm{~K}$ microarray oligonucleotide probe sets within 15q11.2q13.1 targeting distinct interchromosomal duplicons

\begin{tabular}{|c|c|c|}
\hline No. of Probes & Interchromosomal Duplicon Matches & Chromosome Location \\
\hline \multicolumn{3}{|c|}{ BP1 (chr15:18,980,000-20,385,000, NCBI 36; hg18) } \\
\hline 4 & 1 & $13 q 13.2$ \\
\hline 2 & 1 & $2 q 24.3$ \\
\hline 13 & 29 & $13 q 11,18 p 11.21,21 q 11.2,2 q 21.1,22 q 11.1,14 q 11.1$ \\
\hline 3 & 3 & $17 q 11.2$ \\
\hline 1 & 1 & $2 q 14.1$ \\
\hline 11 & 1 & $14 q 11.2$ \\
\hline 6 & 1 & $14 q 32.33$ \\
\hline 1 & 1 & Yp11.32 \\
\hline 2 & 1 & $16 \mathrm{q} 24.2$ \\
\hline 1 & 4 & $16 p 11.2,13 q 31.3$ \\
\hline 2 & 1 & $3 p 22.1$ \\
\hline 1 & 1 & $20 q 13.12$ \\
\hline \multicolumn{3}{|c|}{ BP2 (chr15:20,700,000-21,356,000, NCBI 36; hg18) } \\
\hline 3 & 1 & $16 p 13.12$ \\
\hline 1 & 1 & $13 q 31.3$ \\
\hline 1 & 1 & $16 q 12.2$ \\
\hline \multicolumn{3}{|c|}{ BP3 (chr15:25,941,000-27,286,000, NCBI 36; hg18) } \\
\hline 3 & 4 & $16 p 11.2,13 q 31.3$ \\
\hline 1 & 1 & $3 p 22.3$ \\
\hline 1 & 1 & $5 p 15.31$ \\
\hline
\end{tabular}

The track features from the UCSC genome browser (hg18) of the Agilent SurePrint G3 Human CGH Microarray were analyzed. The Galaxy metaserver was used to extract probe sequences coincident with the non-repeat-masked regions within BP1, BP2 and BP3. Interchromosomal duplicons with complete overlap to the Agilent probe sets were enumerated. In BP1, approximately $73 \%(n=47 / 64)$ of the probes (left column) targeted distinct interchromosomal duplicon loci (middle column) in a chromosome band (right column). Probe targets in intrachromosomal duplicons and repeat-masked sequences were poorly represented in this region (27\%). In BP2, approximately $74 \%(n=14 / 19)$ of the Agilent $244 \mathrm{~K}$ probe sets targeted repeat-masked sequences or intrachromosomal duplicons; however their paralogs were distal to the BP3 region common to AS/PWS deletions. The remaining $26 \%(n=5)$ of the probe sets targeted interchromosomal loci. Probe coverage in BP3 constituted $94 \%(n=74 / 79)$ of both repeat-masked and intrachromosomal duplicon sequences. The remaining probe coverage $(6 \%)$ in BP3 contained targets to both interchromosomal and intrachromosomal duplicon loci that are coincident with BP2. 
Table 2 Number of Affymetrix SNP 6.0 microarray probe sets within 15q11.2q13.1 targeting distinct interchromosomal duplicons

\begin{tabular}{|c|c|c|}
\hline No. of Probes & Interchromosomal Duplicon Matches & Chromosome Location \\
\hline \multicolumn{3}{|c|}{ BP1 (chr15:18,980,000-20,385,000, NCBI 36) } \\
\hline 1 & 1 & $13 q 13.2$ \\
\hline 3 & 1 & $2 \mathrm{q} 24.3$ \\
\hline 1 & 1 & $3 q 29$ \\
\hline 2 & 3 & $22 q 12.2,13 q 12.1$ \\
\hline 4 & 7 & $13 q 12.1,18 p 11.21,21 q 11.2,2 q 21.1,22 q 11.1,14 q 11.1$ \\
\hline 2 & 14 & $18 p 11.2,21 q 11.2,2 q 21.1,22 q 11.1,14 q 11.1$ \\
\hline 1 & 1 & $17 q 11.2$ \\
\hline 1 & 1 & $2 q 14.1$ \\
\hline 4 & 1 & $14 q 11.2$ \\
\hline 1 & 1 & $12 \mathrm{p} 13.31$ \\
\hline 5 & 2 & $16 q 24.2$ \\
\hline 2 & 4 & 1p36.23, Yq11.22 \\
\hline 3 & 1 & $2 \mathrm{p} 24.3$ \\
\hline 3 & 1 & $3 p 22.1$ \\
\hline 7 & 2 & $20 q 13.12$ \\
\hline \multicolumn{3}{|c|}{ BP2 (chr15:20,700,000-21,356,000, NCBI 36) } \\
\hline 8 & 2 & $13 q 31.3$ \\
\hline 3 & 1 & $16 p 13.12$ \\
\hline 3 & 1 & $16 \mathrm{p} 13.12$ \\
\hline \multicolumn{3}{|c|}{ BP3 (chr15:25,941,000-27,286,000, NCBI 36) } \\
\hline 4 & 8 & $13 q 31.3,16 p 11.2$ \\
\hline
\end{tabular}

The probe distribution along 15q11.2 q13 using the Affymetrix SNP 6.0 microarray showed 75\% ( $n=40 / 53$ ) of the probes (left column) in BP1 occur within distinct interchromosomal duplicon loci (middle column) in a given chromosome band (right column). Probes within intrachromosomal duplicons and repeatmasked sequences showed poor representation in this region. Of the 59 probe features targeting SNPs in BP2, 19\% ( $n=11 / 59$ ) were homologous with interchromosomal duplicon intervals and would be expected to cross-hybridize. The remaining $81 \%(n=48 / 59)$ of probe features targeted repeat-masked sequences in intrachromosomal duplicons. Similarly in BP3, the majority of probes occurred within the repeat-masked sequences $(89 \%, n=158 / 177)$, however the remaining probes (11\%) in BP3 contained interchromosomal and intrachromosomal duplicon targets. Probes within BP2 and BP3 are underrepresented relative to adjacent single copy regions.

Table 3 Number of Illumina Beadchip (Human WG) microarray probe sets within 15q11.2q13.1 targeting distinct interchromosomal duplicons

\begin{tabular}{|c|c|c|}
\hline No. Of Probes & Interchromosomal Duplicon Matches & Chromosome Location \\
\hline \multicolumn{3}{|c|}{ BP1 (chr15:20,719,986-22,833,559, GRCh37; hg19) } \\
\hline 1 & 1 & $16 q 12.2$ \\
\hline 1 & 1 & $13 q 13.3$ \\
\hline 7 & 3 & $14 q 32.33$ \\
\hline 1 & 1 & $21 q 11.2,13 q 11,18 p 11.21$ \\
\hline 1 & 6 & $22 q 11.1,2 q 21.1,18 p 11.21,21 q 11.2,14 q 11.2$ \\
\hline 1 & 1 & $16 \mathrm{q} 24.2$ \\
\hline 2 & 1 & $16 q 12.2$ \\
\hline 1 & 1 & $20 q 13.12$ \\
\hline \multicolumn{3}{|c|}{ BP2 (chr15:23,148,559-23,804,907, GRCh37; hg19) } \\
\hline \multicolumn{3}{|c|}{ No probe coverage } \\
\hline \multicolumn{3}{|c|}{ BP3 (chr15:28,267,405-29,498,708, GRCh37; hg19) } \\
\hline 3 & 11 & $16 \mathrm{p} 11.2,22 \mathrm{q} 11.22,19 \mathrm{p} 13.3,1 \mathrm{q} 21.1,22 \mathrm{q} 11.21,1 \mathrm{q} 41,13 \mathrm{q} 31.3$ \\
\hline 2 & 1 & 20p12.1 \\
\hline
\end{tabular}

Probe distribution along 15q11.2q13 with the Illumina Beadchip human whole genome array shows a high proportion of cross-hybridizing probes to SDs in BP1 and BP3, relative to those detecting unique intervals. $75 \%(n=14 / 20)$ of the probes (left column) in BP1 also detect distinct interchromosomal duplicon loci (middle column) in a given chromosome band (right column). In BP3, 50\% ( $n=5 / 10$ ) of the probes hybridized to interchromosomal targets. The remaining probes were found at paralogous loci on chromosome 15 or within repeat-masked regions. Probes within intrachromosomal duplicons ( $\mathrm{n}=5$ ) and repeatmasked sequences $(n=1)$ were poorly represented in BP1 and none were found in BP2. 
The Illumina Beadchip (Human WG) also showed uneven coverage in the $15 \mathrm{q} 11.2 \mathrm{q} 13$ region (Table 3 ). The probes did not cover BP2 at all. BP1 and BP3 were sparsely covered, with 20 probes occurring in BP1-associated SDs and 10 probes in BP3 SDs. This characteristic appears to have been intended in the array design, as poor sensitivity for detection of pathogenic CNVs associated with SDs has been noted [45,46]. Subsequent custom array CGH or MLPA were required to more precisely define these abnormalities. Of those probes detecting these intervals, the majority (19/20 in BP1 and $6 / 10$ in BP3) of these sequences are present in multiple intra or interchromosomal duplicons.

Copy number assessments in regions with two or more highly homologous duplicons can be incorrect due to intrinsic differences relative to balanced copy number. This affects normalization by reducing the dynamic range which can result in misinterpretations when investigating genomic copy number profiles [43,47]. This may explain some of the differences between the breakage intervals delineated in our study and those delineated by BAC and oligonucleotide microarrays. SCFISH may also compliment genome-wide resequencing in SD-rich regions, which are more likely to contain ambiguously mapped reads [48].

\section{Summary}

The chromosomal positions and orientations of genomic probes are essential for defining rearrangements involving SDs. Delineating these rearrangements by SC and LC FISH demonstrates disrupted genes or those whose expression is altered. For disrupted genes whose functions are associated with established phenotypes, the strategy outlined here is likely to be relevant to clinical management and genetic counseling.

\section{Additional material}

Additional file 1: Table S1 - SC and LC primers. Optimized melting temperatures (Tm) and genomic positions (hg18) of the forward and reverse primer pairs.

\begin{abstract}
Acknowledgements
The authors gratefully acknowledge financial support from the University of Western Ontario, Schulich School of Medicine and Dentistry, Canada Foundation for Innovation, Ontario Research Fund (JHMK, PKR), Canada Research Chair and Natural Sciences and Engineering Research Council of Canada (PKR), Ontario Centers of Excellence and Ontario Graduate Scholarship in Science and Technology (WK). The authors also acknowledge Selena Yao for her assistance. We thank the AS families and the London Health Sciences Centre Clinical Cytogenetics laboratory for kindly providing cells and chromosome preparations for FISH probe validation.
\end{abstract}

\section{Author details}

'Department of Biochemistry, University of Western Ontario, Laboratories of Genome Bioinformatics and Genomic Disorders, 1151 Richmond Street,
London, ON, Canada. ${ }^{2}$ Department of Pathology, University of Western Ontario, Laboratories of Genome Bioinformatics and Genomic Disorders, 1151 Richmond Street, London, ON, Canada. ${ }^{3}$ Department of Computer Science, University of Western Ontario, Laboratories of Genome Bioinformatics and Genomic Disorders, 1151 Richmond Street, London, ON, Canada.

\section{Authors' contributions}

PKR, JHMK: conceived and guided the project

PKR: coordinated the study

WK: performed bioinformatic analyses, SC and LC probe development, validation, FISH analyses, cell line culture and chromosome preparations JHMK: provided the AS cell lines and validated cytogenetic preparations WK, JHMK, PKR: wrote the manuscript. All authors have read and approved the final manuscript.

\section{Competing interests}

WK declares no conflict of interest. PKR and JHMK founded Cytognomix Inc. and are the inventors of US Patent Nos. 6,828,097, 7,014,997 and 7,734,424.

Received: 18 April 2011 Accepted: 8 August 2011

Published: 8 August 2011

\section{References}

1. Lupski JR: Genomic disorders: structural features of the genome can lead to DNA rearrangements and human disease traits. Trends Genet 1998, 14:417-422.

2. Lupski JR: Genomic disorders ten years on. Genome Med 2009, 1:42.

3. Mefford HC, Eichler EE: Duplication hotspots, rare genomic disorders, and common disease. Curr Opin Genet Dev 2009, 19:196-204.

4. Knoll JH, Nicholls RD, Magenis RE, Graham JM Jr, Lalande M, Latt SA: Angelman and Prader-Willi syndromes share a common chromosome 15 deletion but differ in parental origin of the deletion. Am J Med Genet 1989, 32:285-290.

5. Knoll JH, Nicholls RD, Magenis RE, Glatt K, Graham JM Jr, Kaplan L, Lalande M: Angelman syndrome: three molecular classes identified with chromosome 15q11q13-specific DNA markers. Am J Hum Genet 1990, 47:149-154.

6. Nicholls RD, Knepper JL: Genome organization, function, and imprinting in Prader-Willi and Angelman syndromes. Annu Rev Genomics Hum Genet 2001, 2:153-175.

7. Christian SL, Fantes JA, Mewborn SK, Huang B, Ledbetter DH: Large genomic duplicons map to sites of instability in the Prader-Willi/ Angelman syndrome chromosome region (15q11.2q13). Hum Mol Genet 1999, 8:1025-1037.

8. Amos-Landgraf JM, Ji Y, Gottlieb W, Depinet T, Wandstrat AE, Cassidy SB, Driscoll DJ, Rogan PK, Schwartz S, Nicholls RD: Chromosome breakage in the Prader-Willi and Angelman syndromes involves recombination between large, transcribed repeats at proximal and distal breakpoints. Am J Hum Genet 1999, 65:370-386.

9. Jiang YH, Wauki K, Liu Q, Bressler J, Pan Y, Kashork CD, Shaffer LG, Beaudet AL: Genomic analysis of the chromosome 15q11.2q13 PraderWilli syndrome region and characterization of transcripts for GOLGA8E and WHCD1L1 from the proximal breakpoint region. BMC Genomics 2008, 9:50.

10. Wang NJ, Liu D, Parokonny AS, Schanen NC: High-resolution molecular characterization of $15 q 11.2 q 13$ rearrangements by array comparative genomic hybridization (array CGH) with detection of gene dosage. Am J Hum Genet 2004, 75:267-281.

11. Locke DP, Segraves R, Nicholls RD, Schwartz S, Pinkel D, Albertson DG, Eichler EE: BAC microarray analysis of $15 q 11.2 q 13$ rearrangements and the impact of segmental duplications. J Med Genet 2004, 41:175-182.

12. Sahoo T, Bacino CA, German JR, Shaw CA, Bird LM, Kimonis V, Anselm I, Waisbren S, Beaudet AL, Peters SU: Identification of novel deletions of $15 q 11$ q13 in Angelman syndrome by array-CGH: molecular characterization and genotype-phenotype correlations. Eur J Hum Genet 2007, 15:943-949.

13. Butler MG, Fischer W, Kibiryeva N, Bittel DC: Array comparative genomic hybridization $(\mathrm{aCGH})$ analysis in Prader-Willi syndrome. Am J Med Genet 2008, 146A:854-860. 
14. Nicholls RD, Knepper JL: Genome organization, function, and imprinting in Prader-Willi and Angelman syndromes. Annu Rev Genomics Hum Genet 2001, 2:153-175

15. Rogan PK, Cazcarro PM, Knoll JH: Sequence-based design of single-copy genomic DNA probes for fluorescence in situ hybridization. Genome Res 2001, 11:1086-1094.

16. Knoll JH, Rogan PK: Sequence-based, in situ detection of chromosomal abnormalities at high resolution. Am J Med Genet 2003, 121A:245-257.

17. Yamada NA, Rector LS, Tsang P, Carr E, Scheffer A, Sederberg MC Aston ME, Ach RA, Tsalenko A, Sampas N, Peter B, Bruhn L, Brothman AR: Visualization of fine scale genomic structure by oligonucleotide-based high-resolution FISH. Cytogenet Genome Res 2011, 132:248-254.

18. Benson DA, Karsch-Mizrachi I, Lipman DJ, Ostell J, Sayers EW: GenBank. Nucleic Acids Res 2011, 39:D32-37.

19. Miller DT, Shen Y, Weiss LA, Korn J, Anselm I, Bridgemohan C, Cox GF, Dickinson H, Gentile J, Harris DJ, Hegde V, Hundley R, Khwaja O, Kothare S, Luedke C, Nasir R, Poduri A, Prasad K, Raffalli P, Reinhard A, Smith SE, Sobeih MM, Soul JS, Stoler J, Takeoka M, Tan WH, Thakuria J, Wolff R, Yusupov R, Gusella JF, et al: Microdeletion/duplication at 15q13.2q13.3 among individuals with features of autism and other neuropsychiatric disorders. J Med Genet 2009, 46:242-248.

20. Blankenberg D, Von Kuster G, Coraor N, Ananda G, Lazarus R, Mangan M, Nekrutenko A, Taylor J: Galaxy: a web-based genome analysis tool for experimentalists. In Curr Protoc Mol Biol. Volume Chapter 19. Edited by: Ausubel F, Brent R, Kingston RE, Moore DD, Seidman JG, Smith JA, Struhl K. New Jersey: John Wiley 2010, Unit 19.10.1-21.

21. Itsara A, Cooper GM, Baker C, Girirajan S, Li J, Absher D, Krauss RM, Myers RM, Ridker PM, Chasman DI, Mefford H, Ying P, Nickerson DA Eichler EE: Population analysis of large copy number variants and hotspots of human genetic disease. Am J Hum Genet 2009, 84:148-161

22. Rozen S, Skaletsky H: Primer3 on the WWW for general users and for biologist programmers. Methods Mol Biol 2000, 132:365-386.

23. Cheng S, Fockler C, Barnes WM, Higuchi R: Effective amplification of long targets from cloned inserts and human genomic DNA. Proc Natl Acad Sci 1994, 91:5695-5699.

24. Knoll JH, Lichter P: In situ hybridization to metaphase chromosomes and interphase nuclei. In Curr Protoc Hum Genet. Volume Chapter 4. Edited by: Haines JL, Korf BR, Morton CC, Seidman CE, Seidman JG, Smith DR. New Jersey: John Wiley 2005, Unit 4.3

25. Pujana MA, Nadal M, Gratacòs M, Peral B, Csiszar K, González-Sarmiento R, Sumoy L, Estivill X: Additional complexity on human chromosome 15q: identification of a set of newly recognized duplicons (LCR15) on 15q11.2q13,15q24, and 15q26. Genome Res 2001, 11:98-111.

26. Ballif BC, Hornor SA, Jenkins E, Madan-Khetarpal S, Surti U, Jackson KE, Asamoah A, Brock PL, Gowans GC, Conway RL, Graham JM Jr, Medne L, Zackai EH, Shaikh TH, Geoghegan J, Selzer RR, Eis PS, Bejjani BA, Shaffer LG: Discovery of a previously unrecognized microdeletion syndrome of 16p11.2-p12.2. Nat Genet 2007, 39:1071-1073.

27. Ji Y, Rebert NA, Joslin JM, Higgins MJ, Schultz RA, Nicholls RD: Structure of the highly conserved $H E R C 2$ gene and of multiple partially duplicated paralogs in human. Genome Res 2000, 10:319-329.

28. Chai JH, Locke DP, Greally JM, Knoll JH, Ohta T, Dunai J, Yavor A, Eichler EE, Nicholls RD: Identification of four highly conserved genes between breakpoint hotspots BP1 and BP2 of the Prader-Willi/Angelman syndromes deletion region that have undergone evolutionary transposition mediated by flanking duplicons. Am J Hum Genet 2003, 73:898-925.

29. Murphy SM, Preble AM, Patel UK, O'Connell KL, Dias DP, Moritz M, Agard D, Stults JT, Stearns T: GCP5 and GCP6: two new members of the human gamma-tubulin complex. Mol Biol Cell 2001, 12:3340-3352.

30. Schenck A, Bardoni B, Moro A, Bagni C, Mandel JL: A highly conserved protein family interacting with the fragile $\times$ mental retardation protein (FMRP) and displaying selective interactions with FMRP-related proteins FXR1P and FXR2P. Proc Natl Acad Sci 2001, 98:8844-8849.

31. Morales J, Hiesinger PR, Schroeder AJ, Kume K, Verstreken P, Jackson FR, Nelson DL, Hassan BA: Drosophila fragile $\times$ protein, DFXR, regulates neuronal morphology and function in the brain. Neuron 2002, 34:961-972.

32. Rainer S, Chai JH, Tokarz D, Nicholls RD, Fink JK: NIPA1 gene mutations cause autosomal dominant hereditary spastic paraplegia (SPG6). Am J Hum Genet 2003, 73:967-971.
33. Apweiler R, Martin MJ, O'Donovan C, Magrane M, Alam-Faruque $Y$, Antunes R, Barrell D, Bely B, Bingley M, Binns D, Bower L, Browne P, Chan WM, Dimmer E, Eberhardt R, Fedotov A, Foulger R, Garavelli J, Huntley R, Jacobsen J, Kleen M, Laiho K, Leinonen R, Legge D, Lin Q, Liu W, Luo J, Orchard S, Patient S, Poggioli D, et al: The Universal Protein Resource (UniProt) in 2010. Nucleic Acids Res 2010, 38:D142-148.

34. Lee ST, Nicholls RD, Bundey S, Laxova R, Musarella M, Spritz RA: Mutations of the $\mathrm{P}$ gene in oculocutaneous albinism, ocular albinism, and PraderWilli syndrome plus albinism. N Engl J Med 1994, 330:529-534.

35. Duffy DL, Montgomery GW, Chen W, Zhao ZZ, Le L, James MR, Hayward NK, Martin NG, Sturm RA: A three-single-nucleotide polymorphism haplotype in intron 1 of OCA2 explains most human eyecolor variation. Am J Hum Genet 2007, 80:241-252.

36. Babatz TD, Kumar RA, Sudi J, Dobyns WB, Christian SL: Copy number and sequence variants implicate APBA2 as an autism candidate gene. Autism Res 2009, 6:359-364.

37. Battaglia A, Novelli A, Bernardini L, Igliozzi R, Parrini B: Further characterization of the new microdeletion syndrome of 16p11.2-p12.2. Am J Med Genet A 2009, 149A:1200-1204.

38. Szafranski P, Schaaf CP, Person RE, Gibson IB, Xia Z, Mahadevan S, Wiszniewska J, Bacino CA, Lalani S, Potocki L, Kang SH, Patel A, Cheung SW, Probst FJ, Graham BH, Shinawi M, Beaudet AL, Stankiewicz P: Structures and molecular mechanisms for common 15q13.3 microduplications involving CHRNA7: benign or pathological? Hum Mutat 2010, 31:840-850.

39. Hannes FD, Sharp AJ, Mefford HC, de Ravel T, Ruivenkamp CA, Breuning MH, Fryns JP, Devriendt K, Van Buggenhout G, Vogels A, Stewart H, Hennekam RC, Cooper GM, Regan R, Knight SJ, Eichler EE, Vermeesch JR: Recurrent reciprocal deletions and duplications of 16p13.11: the deletion is a risk factor for MR/MCA while the duplication may be a rare benign variant. J Med Genet 2009, 46:223-232.

40. Pani AM, Hobart HH, Morris CA, Mervis CB, Bray-Ward P, Kimberley KW, Rios CM, Clark RC, Gulbronson MD, Gowans GC, Gregg RG: Genome rearrangements detected by SNP microarrays in individuals with intellectual disability referred with possible Williams syndrome. PLoS One 2010, 5:e12349.

41. Ou Z, Stankiewicz P, Xia Z, Breman AM, Dawson B, Wiszniewska J, Szafranski P, Cooper ML, Rao M, Shao L, South ST, Coleman K, Fernhoff PM, Deray MJ, Rosengren S, Roeder ER, Enciso VB, Chinault AC, Patel A, Kang SH, Shaw CA, Lupski JR, Cheung SW: Observation and prediction of recurrent human translocations mediated by NAHR between nonhomologous chromosomes. Genome Res 2011, 21:33-46.

42. Shaikh $\mathrm{TH}$ : Oligonucleotide arrays for high-resolution analysis of copy number alteration in mental retardation/multiple congenital anomalies. Genet Med 2007, 9:617-625.

43. Scherer SW, Lee C, Birney E, Altshuler DM, Eichler EE, Carter NP, Hurles ME, Feuk L: Challenges and standards in integrating surveys of structural variation. Nat Genet 2007, 39(Suppl 7):S7-15

44. Neill NJ, Torchia BS, Bejjani BA, Shaffer LG, Ballif BC: Comparative analysis of copy number detection by whole-genome BAC and oligonucleotide array CGH. Mol Cytogenet 2010, 3:11.

45. Knijnenburg J, Oberstein SA, Frei K, Lucas T, Gijsbers AC, Ruivenkamp CA, Tanke HJ, Szuhai K: A homozygous deletion of a normal variation locus in a patient with hearing loss from non-consanguineous parents. J Med Genet 2009, 46:412-417.

46. Kato T, Emi M, Sato H, Arawaka S, Wada M, Kawanami T, Katagiri T, Tsuburaya K, Toyoshima I, Tanaka F, Sobue G, Matsubara K: Segmental copy-number gain within the region of isopentenyl diphosphate isomerase genes in sporadic amyotrophic lateral sclerosis. Biochem Biophys Res Commun 2010, 402:438-442.

47. Carter NP: Methods and strategies for analyzing copy number variation using DNA microarrays. Nat Genet 2007, 39(Suppl 7):S16-21.

48. Alkan C, Kidd JM, Marques-Bonet T, Aksay G, Antonacci F, Hormozdiari F, Kitzman JO, Baker C, Malig M, Mutlu O, Sahinalp SC, Gibbs RA, Eichler EE: Personalized copy number and segmental duplication maps using nextgeneration sequencing. Nat Genet 2009, 41:1061-1067.

doi:10.1186/1755-8166-4-15

Cite this article as: Khan et al:: Context-based FISH localization of genomic rearrangements within chromosome 15q11.2q13 duplicons. Molecular Cytogenetics 2011 4:15. 